\title{
Modulation and Compensation of the mRNA Expression of Energy Related Transporters in the Brain of Glucose Transporter 1-Deficient Mice
}

\author{
Sumio Ohtsuki, ${ }^{a, b, c}$ Tazuru Kikkawa, ${ }^{a}$ Satoko Hori, ${ }^{a, b, c}$ and Tetsuya Terasaki ${ }^{*, a, b, c}$ \\ ${ }^{a}$ Department of Molecular Biopharmacy and Genetics, Graduate School of Pharmaceutical Sciences, Tohoku University; \\ ${ }^{b}$ New Industry Creation Hatchery Center, Tohoku University; Aoba, Aramaki, Aoba-ku, Sendai 980-8578, Japan: and \\ ${ }^{c}$ SORST and CREST of Japan Science and Technology Agency; Japan. \\ Received March 13, 2006; accepted May 16, 2006; published online May 18, 2006
}

Facilitative glucose transporter 1 (GLUT1) is the molecule responsible for the entry of glucose into the brain, and its mutation is known as GLUT1 deficiency syndrome (GLUT1DS) in humans. To clarify the effect of GLUT1 gene deficiency, we have produced GLUT1-deficient mice, and investigated the developmental expression of GLUT1, monocarboxylate transporter 1 (MCT1) and MCT2 in the brains of these mice. Since the homozygotes were found to be embryonically lethal and the heterozygotes exhibited no abnormalities, GLUT1deficiency was examined using heterozygote mice. GLUT1 deficiency did not significantly affect the mRNA levels of GLUT1 at P0, P7 and in adults, or the levels of MCTs at P7, P14 and in adults. The GLUT1 level at P14 was reduced by 46.9\%, although this was not statistically significant. The MCTs levels at P0 were increased about 2.0 -fold in the deficient mice compared with the wild type. Furthermore, at P0, GLUT1 mRNA levels in wild type females were 1.91-fold higher than in wild type males. These results suggest that GLUT1 deficiency affects GLUT1 mRNA expression in the infant brain, and that of MCT1 and MCT2 in the neonatal brain. Furthermore, a compensatory effect of GLUT1 expression was observed in the brain of adult deficient mice. These effects of GLUT1 deficiency in the brain provide a molecular basis to assist in our understanding of the symptoms of GLUT1DS.

Key words glucose transporter 1 (GLUT1); deficient syndrome; monocarboxylate transporter; OmniBank; mRNA expression; gene compensation

Facilitative glucose transporter 1 (GLUT1/SLC2A1) is a key molecule governing the cerebral glucose supply from the circulating blood, since GLUT1 is expressed abundantly on the abluminal and luminal membrane of the capillary endothelial cells which form the blood-brain barrier (BBB) ${ }^{1}{ }^{1}$ The disease associated with a heterozygotic mutation in the GLUT1 gene is known as GLUT1 deficiency syndrome (GLUT1DS). ${ }^{2)}$ GLUT1DS involves hypoglycorrhachia without hypoglycemia. ${ }^{3)}$ The patients exhibit clinical features associated with central nervous system (CNS) function, including infantile seizures, delayed neurological development, incoordination and spasticity. ${ }^{3)}$ This clinical evidence supports the hypothesis that glucose transport by GLUT1 is a critical process for the provision of cerebral energy to develop and maintain CNS function.

Although the symptoms of GLUT1DS provide important evidence to help us understand the function of GLUT1 in the brain, the molecular basis of the effects of GLUT1 gene deficiency remains largely unknown. The effect of GLUT1 deficiency was expected to changed during development, since the existence of a compensatory mechanism has been reported following heterozygous knockout of the GLUT1 gene in embryonic stem cells. ${ }^{4}$ Furthermore, the brain capillary endothelial cells express monocarboxylate transporter 1 (MCT1) to allow the entry of lactate and ketone bodies into the brain as a secondary energy source. ${ }^{5,6}$ Indeed, for GLUT1DS patients, a ketogenic diet is effective in controlling seizures that are resistant to anti-epileptic drug treatment. ${ }^{7)}$ Neurons have also been reported to express MCT2, which may mediate the entry of lactate as an energy source for neurons. ${ }^{8)}$ Thus, the link between GLUT1 expression and MCTs expression can be considerable. The effect of GLUT1 gene deficiency on the expression of MCTs, as well as that of
GLUT1, in the brain needs to be clarified in order to understand the regulation of energy supply to the brain.

The purpose of the present study was to generate GLUT1 deficient mice by gene-trapping, and to clarify the effect of GLUT1 gene deficiency on the developmental expression of GLUT1, MCT1 and MCT2 in the brain.

\section{MATERIALS AND METHODS}

Generation of GLUT1-Deficient Mice All experiments were approved by the Animal Care Committee, Graduate School of Pharmaceutical Sciences, Tohoku University. GLUT1-deficient mice were generated from the Gene-Trapping embryonic stem cell bank (OmniBank) of Lexicon Genetics Incorporated (Woodlands, TX, U.S.A.). ${ }^{9,10)}$ The trapping vector contains a splicing acceptor (SA) followed by neomycin resistance (neo) gene with a polyadenylation signal (Fig. 1B). The SA in the vector traps the endogenous splicing, leading to a truncated mRNA. The vector also contains a promoter of mouse phosphoglycerate kinase $(P g k)$ gene followed by murine Bruton's tyrosine kinase $(B t k)$ gene with splicing donor (SD). Splicing from this SD to the exons downstream of the insertion produced a fusion mRNA. The genomic and cDNA sequence around the insertion site was registered in the OmniBank database.

ES cells in which the trapping vector was inserted in intron 1 of the GLUT1 gene were selected and the mice produced as described previously. ${ }^{9)}$ The chimeric mice were bred to $\mathrm{C} 57 \mathrm{BL} / 6 \mathrm{~J}$. The generated mouse strain was maintained by mating between heterozygotes. Mating was conducted by placing male and female mice in the same box for $13 \mathrm{~h}$ from 9 p.m. to 10 a.m., and the time separating males and females was counted as embryonic day 0.5. Postnatal 
days were counted from the date of birth when the adult mice were 8-16 weeks old. Plasma glucose levels were measured using a Glucose Assay kit (Sigma; St. Louis, MO, U.S.A.)

Genotyping of the GLUT1-Deficient Mice The genotyping of all offspring was analyzed by PCR of genomic DNA from the tail using a DNeasy Tissue Kit (QIAGEN; Valencia, CA, U.S.A.). The primers shown in Fig. 1 are as follows: $a$, 5'-GGGCCACAGTGATAGAGATAGAATG-3'; $b$, 5'-GTGTGACTTTAGGAAACTTACTTCCC-3'; $c$, 5' AAATGGCGTTACTTAAGCTAGCTTGC-3'. The PCR was conducted with the following program: 1 cycle of $94^{\circ} \mathrm{C}$ for $2 \mathrm{~min}, 30$ cycles of $94^{\circ} \mathrm{C}$ for $30 \mathrm{~s}, 60^{\circ} \mathrm{C}$ for $30 \mathrm{~s}, 72^{\circ} \mathrm{C}$ for $1 \mathrm{~min}$ and a final elongation of $72^{\circ} \mathrm{C}$ for $10 \mathrm{~min}$. The expected size of the product with primers $(a+b)$ is $488 \mathrm{bp}$, and that with $(c+d)$ is $504 \mathrm{bp}$.

Analysis of Mutation by RT-PCR Total RNA was prepared from the brain using TRIzol reagent (Invitrogen, Carlsbad, CA, U.S.A.). Reverse-transcription was performed with oligo-dT primer. The primers for RT-PCR analysis shown in Fig. 1 are as follows: $d$, 5'-CTAGAGCTTCGAGCGCAGCGC-3'; $e, 5^{\prime}$-AGGCCAACAGGTTCATCATC-3'; $f$, 5'-TTGGTGGTCGAATGGGCAGGT-3'. Primers $d$ and $e$ were designed from the nucleotide sequence in exon 1 and 4 of the GLUT1 gene, respectively. PCR was conducted with the following program: 1 cycle at $94{ }^{\circ} \mathrm{C}$ for $2 \mathrm{~min}, 30$ cycles at $94{ }^{\circ} \mathrm{C}$ for $30 \mathrm{~s}, 60^{\circ} \mathrm{C}$ for $30 \mathrm{~s}, 72^{\circ} \mathrm{C}$ for $1 \mathrm{~min}$ and a final elongation at $72^{\circ} \mathrm{C}$ for $10 \mathrm{~min}$. The expected size of the product with primers $(d+e)$ is $335 \mathrm{bp}$. PCR products were subcloned and sequenced using a DNA sequencer (CEQ2000XL; Beckman Coulter, Fullerton, CA, U.S.A.).

Quantitative Real-Time PCR Analysis Single-strand cDNA was synthesized from total RNA $(1 \mu \mathrm{g})$ by reverse transcription using oligo dT primer. According to the manufacturer's protocol, quantitative real-time PCR was performed using an ABI PRISM 7700 sequence detector system (Applied Biosystems; Foster City, CA, U.S.A.) with a $2 \mathrm{X}$ SYBR Green PCR master mix (Applied Biosystems), reverse transcribed cDNA, and gene-specific primers. To quantify the amount of target mRNA in the samples, a standard curve was prepared for each run using the plasmid containing the target gene. The primers $d$ and $e$ shown in Fig. 1A were used for GLUT1. The sequences of the specific primers were as follows: 5'-GCCTGAGCAAGTCAAGCTAGAAA-3' and 5'-CATTTGCAACAACAGAAGCAGC-3' for MCT1; 5'CCAAAGCAAGAGCTCATCTACA-3' and 5'-CAGGCCGGCTGCCATAGTT-3' for MCT2; 5'-TTTGAGACCTTCAACACCCC-3' and 5'-ATAGCTCTTCTCCAGGGAGG-3' for $\beta$-actin. PCR was conducted through 1 cycle at $95^{\circ} \mathrm{C}$ for $10 \mathrm{~min}, 40$ cycles at $95^{\circ} \mathrm{C}$ for $30 \mathrm{~s}, 60^{\circ} \mathrm{C}$ for $1 \mathrm{~min}$, and $72{ }^{\circ} \mathrm{C}$ for $1 \mathrm{~min}$. The mRNA levels were normalized relative to that of $\beta$-actin. The statistical significance of differences among means of several groups was determined by one-way analysis of variance (ANOVA), and an unpaired, two-tailed Student's $t$-test was used to determine the significance of differences between two groups. PCR products were subcloned and sequenced using a DNA sequencer (CEQ2000XL; Beckman Coulter).

\section{RESULTS}

Mutation of GLUT1 Gene by the Trapping Vector The mutation of the GLUT1 gene by insertion of the trapping vector was examined by genomic PCR and RT-PCR analysis with the primer sets shown in Figs. $1 \mathrm{~A}$ and B. With the primers $(a+b)$ for detecting the wild type allele, bands at the expected size were detected in wild type and hyterozygotic mice (Fig. 1C). In contrast, with the primers $(c+b)$ for the mutated allele, the expected band was detected in heterozygotes but not in wild type animals (Fig. 1C). This indicates that the trapping vector was inserted in the expected site in intron 1 of the GLUT1 gene.

RT-PCR was conducted using total RNA isolated from the brain of heterozygotic mice (Fig. 1D). A band was detected with primers $(d+e)$ at the expected size, and the nucleotide sequence of the product completely matched that of the corresponding region of the GLUT1 cDNA. A band at $500 \mathrm{bp}$ was detected with primers $(d+f)$, and the sequence of the product contains a fused sequence of the GLUT1 gene and neo gene. This result demonstrates that the inserted trapping vector truncated the GLUT1 mRNA.

Phenotypes of the GLUT1-Deficient Mice Mating with heterozygotic mice, wild type and heterozygotic pups was carried out, however no homozygote was obtained during the entire study. The genotyping was conducted for the embryos from embryonic day 12 to day 18, and no homozygotic embryo was detected in a total of 45 embryos. Therefore, the
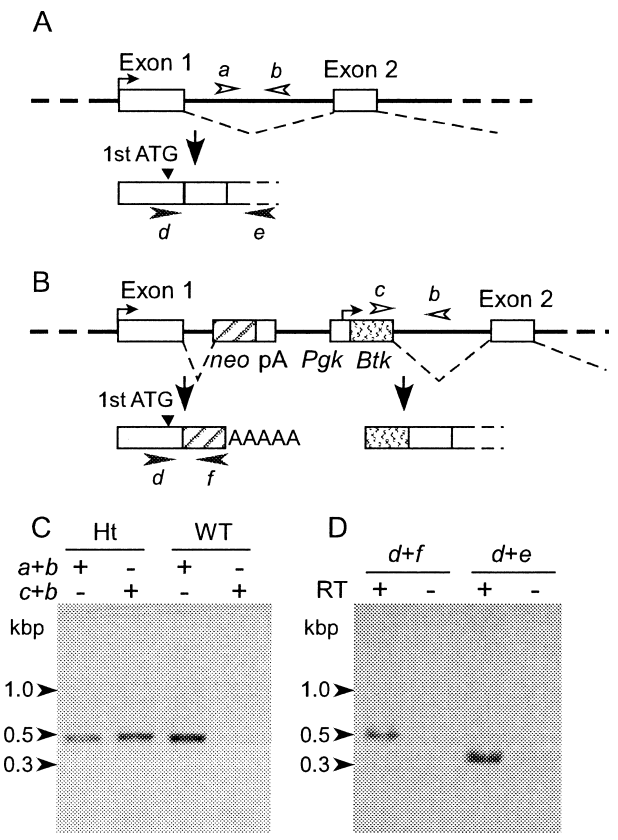

Fig. 1. Generation of GLUT1-Deficient Mice

(A, B) The locations of primers for genomic PCR (open arrow heads) and RT-PCR (closed arrow heads) in the wild type gene (A) and the trapped gene (B). Primers $a$ and $b$ flank the genomic insertion site of the gene-trapping vector in intron 1 of the GLUT-1 gene and amplify a product for the wild type allele. Primer $c$ was used in conjunction with primer $b$ to specifically amplify the mutated allele. Primers $d$ and $e$ were designed from the GLUT1 cDNA sequence of exon 1 and exon 4, respectively, for the wild type. Primer $f$ was annealed with neo for amplifying the flanked gene combined with primer $d$. (C) Genomic PCR of wild type (WT) and heterozygotic mice (Ht) with the primer sets of $a$ and $b(a+b)$ for the wild type allele or $c$ and $b(c+b)$ for the mutated allele. (D) RT-PCR analysis in heterozygotic mouse brain with $(+)$ or without $(-)$ reversetranscription. Total RNA was isolated from the brain. neo, neomycin resistance gene; pA, polyadenylation signal; $P g k$, phosphoglycerate kinase gene promoter; Btk, Bruton's tyrosine kinase gene. 
homozygotic embryos would be embryonically lethal before embryonic day 12, and the heterozygotic mice were used as GLUT1-deficient mice in later studies.

No obvious abnormalities in the behavior and reproductive ability were observed in the GLUT1-deficient mice. The body weights of the GLUT1-deficient and wild type mice were $28.1 \pm 1.2 \mathrm{~g}$ and $25.2 \pm 0.5 \mathrm{~g}$ in males (11 weeks), and $17.4 \pm 0.5 \mathrm{~g}$ and $15.9 \pm 0.6 \mathrm{~g}$ in females (6 weeks), respectively (the mean \pm S.E.M., $n=3$ ). The GLUT1-deficient mice tended to be heavier, although the difference was not significant. Furthermore, there were no significant differences in brain weight and plasma glucose levels between the deficient and wild type mice (data not shown).

Expression of GLUT1 mRNA in the Brain of GLUT1Deficient Mice The mRNA expression levels of GLUT1 in the brain were examined at postnatal day 0,7 and 14 (P0, P7 and P14), and in adults. As shown in Fig. 2A, GLUT1 mRNA levels in the brain were the lowest at P7 and increased to adult levels in the GLUT1-deficient or wild type mice. While no statistically significant difference was observed at each age, the GLUT1 mRNA levels at P14 in the deficient mice were reduced by $46.9 \%$ compared with those in wild type mice ( $n=21$ and 7 for wild type and heterozygotes, respectively).

There was no significant difference between males and females with regard to the GLUT1 mRNA levels in the brain at each age except P0. At P0, in wild type mice, the GLUT1 mRNA level in the brain of females was 1.91-fold greater
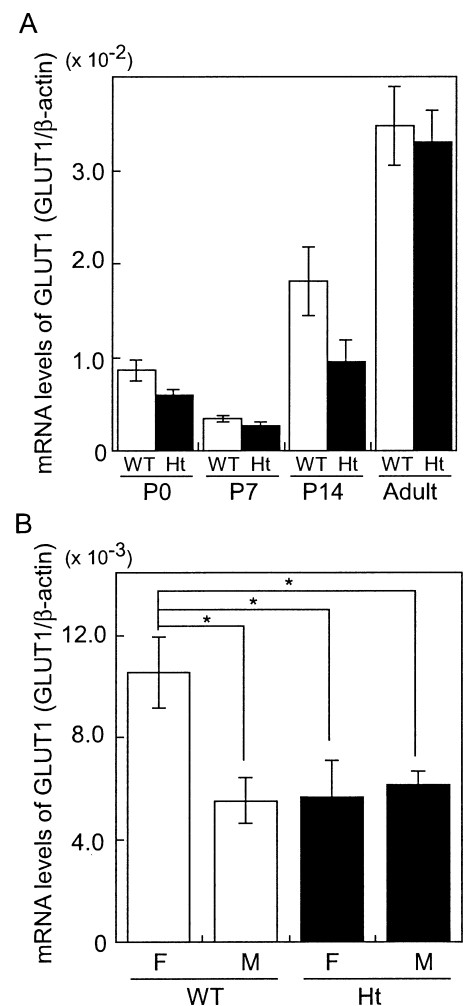

Fig. 2. Expression of GLUT1 mRNA in the Brain of GLUT1-Deficient Mice

(A) Developmental expression of GLUT1 gene in the brain of heterozygotic mice (close bar) and wild type mice (open bar) at postnatal day 0, 7 and 14 (P0, P7 and P14, respectively) and in the adult. (B) Comparison of GLUT1 mRNA expression at P0 in the brain of female $(\mathrm{F})$ and male $(\mathrm{M})$ mice. WT, wild type mice; Ht, heterozygotic mice. Expression of GLUT1 mRNA was normalized by that of $\beta$-actin. Each bar represents the mean \pm S.E.M. (A, $n=6-21 ; \mathrm{B}, n=4-8)$. $* p<0.05$, significant difference. than that of males (Fig. 2B). In the deficient mice, males and females had similar mRNA levels to the wild type males.

Developmental Expression of MCT1 and MCT2 in the Brain of GLUT1-Deficient Mice MCT1 mRNA expression in the brain increased with development, and reached a maximum at P14 (Fig. 3A). In adults, MCT1 mRNA expression was suppressed. In contrast, MCT2 mRNA expression was low from $\mathrm{P} 0$ to $\mathrm{P} 14$, and increased in adults (Fig. 3B). At P0, MCT1 and MCT2 mRNA levels in the brain of the deficient mice were 1.96-fold and 2.00-fold greater than that of wild type mice. No significant difference between males and females was detected with regard to the MCT1 and MCT2 mRNA levels at P0 (Fig. 3C), P7 and P14, and in adults (data not shown).

\section{DISCUSSION}

The present study is the first demonstration of the individ-
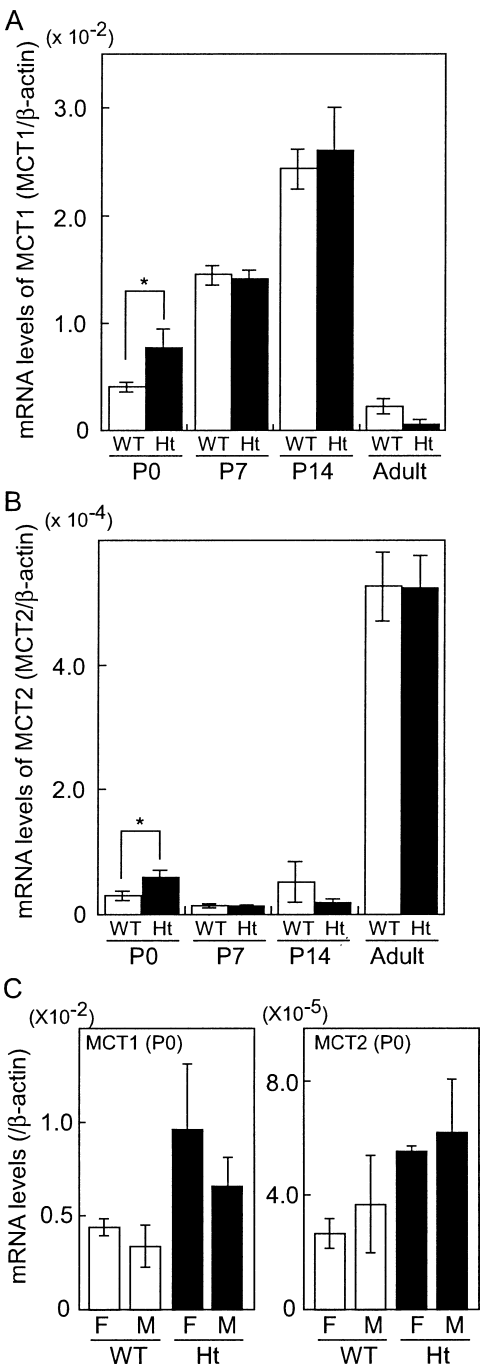

Fig. 3. Developmental Expression of MCT1 (A) and MCT2 (B) mRNA in the Brain

The mRNA expression was normalized by that of $\beta$-actin in the brain of heterozygotic mice (close bar) and wild type mice (open bar) at postnatal day 0, 7 and 14 (P0 $\mathrm{P} 7$ and P14, respectively) and in the adult. Each bar represents the mean \pm S.E.M. (A $n=6-22 ; \mathrm{B}, n=3-17) . * p<0.05$, significant difference. (C) mRNA levels of MCT1 (left panel) and MCT2 (right panel) in males and females at P0. Each bar represents the mean \pm S.E.M. (MCT1, $n=4-16$; MCT2, $n=3-8$ ). WT, wild type mice; Ht, heterozygotic mice; F, females; M, males. 
ual characteristics of GLUT1-deficient mice following knock out of the GLUT1 gene. Heilig et al. (2003) have described GLUT1-deficient transgenic mice produced by antisense treatment, and the expression of antisense from the transgene varied from one tissue to another. In contrast, the insertion of the trapping vector truncated GLUT1 mRNA resulting in translation of the first 6 amino acids in the intracellular region (Fig. 1). Therefore, in the deficient mice reported in this study, the GLUT1 gene expression is completely absent in homozygotes, and phenotypes of heterozygotes reflect a lack of the GLUT1 gene on single alleles in the whole body. In this study, heterozygotes were used to investigate GLUT1 deficiency, since the homozygotes were found to be embryonically lethal.

The deficiency in the GLUT1 gene was expected to attenuate the expression and function of GLUT1 at the BBB, leading to a suppression of the supply of glucose to the brain. However, the heterozygotes showed no obvious abnormalities compared with the wild type, suggesting the existence of a compensatory mechanism to rescue the gene deficiency in knock out mice. The function of GLUT is controlled by complex processes, such as transcriptional and post-transcriptional regulation and subcellular localization. ${ }^{11,12)}$ In addition, there is a report that GLUT1 is also expressed in astrocytes with a smaller molecular size than in brain capillary endothelial cells. ${ }^{13)}$ As the first study to clarify the effect of GLUT1 gene deficiency on brain GLUT1 expression during the developmental stages, the effect was initially assessed by mRNA expression using RT-PCR analysis.

The GLUT1 mRNA expression in the brain of both wild type and GLUT1-deficient mice was reduced at P7 and increased in adults (Fig. 2A). This developmental expression is similar to that reported in rabbit brain capillary endothelial cells and rat hippocampus. ${ }^{14,15)}$ GLUT1 gene deficiency does not affect GLUT1 mRNA expression in the adult brain, indicating that GLUT1 mRNA expression is maintained at normal levels in the deficient mouse brain by a compensatory mechanism, such as the enhancement of the transcription rate and/or mRNA stability.

At P14, the GLUT1 expression was suppressed in the deficient mice compared with the wild type, although this difference was not statistically significant (Fig. 2A). This result indicates the possibility that GLUT1 expression in the brain is reduced during infancy in the brain of GLUT1DS patients. The large individual difference at P14 is probably due to the fact that the induction of GLUT1 in the brain during development varied depending on the individual growth state.

These changes in GLUT1 mRNA expression observed in the deficient mice correlate with several clinical features of GLUT1 DS. The gene compensation of GLUT1 in adult deficient mice implies that adult patients with GLUT1DS express normal levels of GLUT1 in the brain, and this would explain the improvement in symptoms with maturity. ${ }^{16,17)}$ The attenuation of brain GLUT1 mRNA levels in the deficient mice at P14 indicates the possibility that the GLUT1 function at the BBB of infant GLUT1 DS patients is suppressed.

The present study demonstrated a gender difference in the GLUT1 mRNA expression in the brain of neonatal mice (Fig. 2B). This gender difference in GLUT1 expression disappeared in GLUT1 deficient mice which had the same levels as wild type males. Although the physiological signifi- cance of this difference is unknown, an interesting population-based study of the risk of neonatal seizures has been reported. ${ }^{18)}$ This study showed that the risk of seizures in preterm male infants was 1.8 -fold higher than that in preterm female infants. The lower expression of GLUT1 in the neonatal male brain is likely to correlate with the higher risk of neonatal seizures.

MCT1 is expressed in brain capillary endothelial cells, and the expression of MCT1 in the brain and brain uptake rate of lactate is increased during the suckling period. ${ }^{19,20)}$ It is thought that the induction of a lactate uptake system in the brain is to allow the use of the lactate in milk. In the deficient mice, MCT1 expression in the brain reached its highest levels at P14, the same levels as in the wild type (Fig. 3A). Therefore, during the infantile period, MCT1 is suggested to be expressed in sufficient quantity to supply lactate and ketone bodies to the brain to bypass the GLUT1 defect. In contrast to MCT1, MCT2 mRNA expression reached its highest levels in the adult brain (Fig. 3B). This suggests the possibility that transporter(s) other than MCT2 are involved in the entry of lactate and ketone bodies into neurons during infancy.

MCT1 and MCT2 expression in the neonatal brain is about 2.0-fold higher in deficient mice than in wild type animals (Fig. 3C). Therefore, GLUT1 deficiency would influence the expression and function of GLUT1, MCT1 and MCT2 in the brain of late embryos and neonates in GLUT1DS. Although the function of these energy-related transporters in the brain of late embryos and neonates remains to be clarified, these results raise the possibility that GLUT1 deficiency influences the neural development of late embryos and neonates.

Although infantile seizures are one of the typical clinical features of GLUT1DS, the heterozygotes showed no obvious abnormalities compared with the wild type. This indicates one of the difficulties in producing GLUT1DS model animals and suggests differences in energy supply between humans and mice. There are two likely reasons for this. One is a difference in the regulation mechanism, i.e. GLUT1 gene deficiency affects the GLUT1 function in the brain more markedly in humans than in mice. It is also possible that the rates and/or period of gene induction of GLUT1 and MCT1 during development are different between human and mouse brain. The other reason involves environmental differences including dietary intake. Since a ketogenic diet is effective in improving the symptoms of GLUT1DS, not only the glucose supply rate to the brain but also the serum levels of ketone bodies could be important for the development of the phenotypes of GLUT1 gene deficiency. Therefore, further ethological and histological studies with dietary and environmental controls are necessary to clarify the neurological symptoms of GLUT1 deficient mice.

In conclusion, this is the first report of the production and characterization of GLUT1-deficient mice by gene trapping. GLUT1 gene deficiency attenuates the mRNA expression of GLUT1 in the infantile brain, and induces that of MCT1 and MCT2 in the neonatal brain. Furthermore, the present study shows the compensatory effect of GLUT1 expression in the brain of adult deficient mice. As described earlier, the control of GLUT1 function involves multiple processes. Therefore, based on the present evidence, further analysis of protein ex- 
pression, localization and the functions of energy-related transporters, such as brain uptake of glucose, in the brain of deficient mice is necessary to provide a molecular basis for the regulation of energy supply to the brain and the symptoms of GLUT1DS.

Acknowledgments This study was supported, in part, by a Grant-in-Aid for Scientific Research and a grant for the 21st Century Center of Excellence (COE) Program Special Research Grant from the Japan Society for the Promotion of Science, and the Industrial Technology Research Grant Program from New Energy and the Industrial Technology Development Organization (NEDO) of Japan.

\section{REFERENCES}

1) Pardridge W. M., Boado R. J., Farrell C. R., J. Biol. Chem., 265, 18035-18040 (1990).

2) De Vivo D. C., Trifiletti R. R., Jacobson R. I., Ronen G. M., Behmand R. A., Harik S. I., N. Engl. J. Med., 325, $703-709$ (1991).

3) Pascual J. M., Wang D., Lecumberri B., Yang H., Mao X., Yang R., De Vivo D. C., Eur. J. Endocrinol., 150, 627-633 (2004).

4) Heilig C., Brosius F., Siu B., Concepcion L., Mortensen R., Heilig K., Zhu M., Weldon R., Wu G., Conner D., Am. J. Pathol., 163, 18731885 (2003).

5) Leino R. L., Gerhart D. Z., Drewes L. R., Brain Res. Dev. Brain Res., 113, 47-54 (1999).

6) Terasaki T., Takakuwa S., Moritani S., Tsuji A., J. Pharmacol. Exp. Ther., 258, 932-937 (1991).
7) Klepper J., Diefenbach S., Kohlschutter A., Voit T., Prostaglandins Leukot Essent Fatty Acids, 70, 321-327 (2004).

8) Rafiki A., Boulland J. L., Halestrap A. P., Ottersen O. P., Bergersen L., Neuroscience, 122, 677-688 (2003).

9) Zambrowicz B. P., Abuin A., Ramirez-Solis R., Richter L. J., Piggott J., BeltrandelRio H., Buxton E. C., Edwards J., Finch R. A., Friddle C. J., Gupta A., Hansen G., Hu Y., Huang W., Jaing C., Key B. W., Jr., Kipp P., Kohlhauff B., Ma Z. Q., Markesich D., Payne R., Potter D. G., Qian N., Shaw J., Schrick J., Shi Z. Z., Sparks M. J., Van Sligtenhorst I., Vogel P., Walke W., Xu N., Zhu Q., Person C., Sands A. T., Proc. Natl. Acad. Sci. U.S.A., 100, 14109-14114 (2003).

10) Zambrowicz B. P., Friedrich G. A., Buxton E. C., Lilleberg S. L., Person C., Sands A. T., Nature (London), 392, 608-611 (1998).

11) Boado R. J., Pardridge W. M., Brain Res. Mol. Brain Res., 59, 109113 (1998).

12) Sanchez-Alvarez R., Tabernero A., Medina J. M., J. Neurochem., 89, 703-714 (2004).

13) Yu S., Ding W. G., Brain Res., 797, 65-72 (1998).

14) Dwyer K. J., Pardridge W. M., Endocrinology, 132, 558 - 565 (1993).

15) Vannucci S. J., Simpson I. A., Am. J. Physiol. Endocrinol. Metab., 285, E1127-1134 (2003).

16) Brockmann K., Wang D., Korenke C. G., von Moers A., Ho Y. Y., Pascual J. M., Kuang K., Yang H., Ma L., Kranz-Eble P., Fischbarg J., Hanefeld F., De Vivo D. C., Ann. Neurol., 50, 476- 485 (2001).

17) Gordon N., Newton R. W., Brain Dev., 25, 477-480 (2003).

18) Saliba R. M., Annegers F. J., Waller D. K., Tyson J. E., Mizrahi E. M., Am. J. Epidemiol., 154, 14-20 (2001).

19) Cremer J. E., Cunningham V. J., Pardridge W. M., Braun L. D., Oldendorf W. H., J. Neurochem., 33, 439-445 (1979).

20) Pellerin L., Pellegri G., Martin J. L., Magistretti P. J., Proc. Natl. Acad. Sci. U.S.A., 95, 3990-3995 (1998). 